\title{
MASSIVE GAUGE AXION FIELDS
}

\author{
P. J. Arias \\ Grupo de Campos y Partículas, Departamento de Física, Facultad de \\ Ciencias, Universidad Central de Venezuela, \\ AP 47270, Caracas 1041-A, Venezuela. \\ A. Khoudeir \\ Centro de Astrofísica Teórica, Departamento de Física, Facultad de \\ Ciencias, Universidad de los Andes, \\ Mérida, 5101, Venezuela.
}

\begin{abstract}
A gauge invariant formulation for the massive axion is considered. The axion acquires mass through a topological term which couples a (pseudo)scalar and a third rank antisymmetric tensor. Duality, local and canonical equivalences with the non-gauge invariant proposal are established. The supersymmetric version of the gauge invariant model is constructed.
\end{abstract}

It is well known that different theories can be related by duality transformations and their classical and quantum properties are equivalent. In four dimensions a massless (pseudo)scalar field: the axion, is dual to the antisymmetric field $B_{m n}$ (only if derivative couplings are considered). This fact is a particular case of the general duality between $p$ and $D-p-2$ forms in $D$ dimensions. In this sense, it is known that non-perturbative effects break the Pecci-Quinn (PQ) symmetry of the axion, giving mass to it. Then, a natural question arises, concernig about what happens with the duality symmetry. This item was an enigma until two independent approaches were developed recently [1], [2]. In these references the authors show that, though the PQ symmetry is broken, duality symmetry still remains. The $B_{m n}$ field is replaced by a massive antisymmetric field of third rank $C_{m n p}$. A characteristic feature of this duality is the lost of abelian gauge invariance for the new antisymmetric field. The duality now corresponds to a particular case of the usual duality between massive $p$ and $D-p-1$ forms in $\mathrm{D}$ dimensions.

The conection between a massive (pseudo)scalar field, $\phi$, and a massive antisymmetric field, $C_{m n p}$ has been considered before in different contexts 
[3] [1] [5] [6]. In this letter, we will show that a gauge invariant model, which involves a topological coupling, considered in reference [4], in the context of the $U(1)$ problem, is locally equivalent to the non gauge invariant proposal for the massive axion. This equivalence is analogous to that between the "self-dual" [0] and topologically massive [8] models in three dimensions, in the same manner as the equivalence between the Proca and the topologically massive gauge invariant theories in four dimensions [9] [10]. In the preceding examples, in three and four dimensions, the connection occurs between a gauge sensitive and a gauge invariant model. It has been shown that these models are locally equivalent and one of them is a gauge fixed version of the other [11][9]. Nevertheless the global equivalence depends on the cohomological structure of the base manifold [12] 9] [13] [14]. For the models that we compare in this letter we will face this dual equivalence in several ways. One of them, described below by $I_{M 1}$, is a first order version of the massive (pseudo)scalar model. The other model, described below by $I_{M 2}$, is gauge invariant. We will note that the space of solutions of both models differ in a topological sector that can be gauged out on trivial manifolds. The presence of this topological sector appears explicitly in the partiton function of the gauge invariant model as a topological factor related with the cohomological structure of the base manifold. We will prove (canonically) that one of the models is a gauge fixed version of the gauge invariant one. In particular, we will see the equivalence through the existence of a first order master action, that will allow us to consider global aspects and interactions. Finally we will construct a supersymmetric formulation of the gauge invariant action of the massive axion.

An illustrative model for the massive axion is given by the following master action [5] [15]

$$
I=<\frac{1}{2} v_{m} v^{m}+\phi \partial_{m} v^{m}-\frac{1}{2} m^{2} \phi^{2}>
$$

where $v_{m}$ is a vector field and $\phi$ is a scalar field $(<>$ denotes integration in four dimensions). Eliminating the field $v_{m}$ through its equation of motion $\left(v_{m}=\partial_{m} \phi\right)$, the action for a massive scalar is obtained, while using the equation of motion of the scalar field $\phi\left(\phi=\frac{1}{m^{2}} \partial_{m} v^{m}\right)$, we have

$$
I_{v}=\frac{1}{2}<v_{m} v^{m}+\frac{1}{m^{2}}\left(\partial_{m} v^{m}\right)^{2}>
$$


The propagator corresponding to the field $v_{m}$ is $\eta_{m n}-\frac{k_{m} k_{n}}{k^{2}+m^{2}}$, which is just equal to those discussed in [1]. If we introduce the dual of the vector field: $v^{m}=\frac{1}{3 !} m \epsilon^{m n p q} C_{n p q}$, we have the following master action

$$
I_{M 1}=<-\frac{1}{2.3 !} m^{2} C^{m n p} C_{m n p}+\frac{1}{3 !} m \epsilon^{m n p q} \phi \partial_{m} C_{n p q}-\frac{1}{2} m^{2} \phi^{2}>,
$$

from which duality is easily infered. To see this, we can use its equations of motion

$$
\begin{gathered}
m C^{m n p}-\epsilon^{m n p q} \partial_{q} \phi=0, \\
m \phi-\frac{1}{3 !} \epsilon^{m n p q} \partial_{m} C_{n p q}=0,
\end{gathered}
$$

to eliminate $C_{m n p}$ (or $\phi$ ), thus obtaining the action for a massive scalar field (or the massive antisymmetric field $C_{m n p}$ ). In any case, there is not gauge invariance at all. We can ask whether there really exist a gauge invariant theory compatible with a massive term for the axion field. The answer is positive, and the action is

$$
I_{M 2}=<-\frac{1}{2} \partial_{m} \phi \partial^{m} \phi-\frac{1}{2.4 !} G_{m n p q} G^{m n p q}-\frac{m}{3 !} \epsilon^{m n p q} C_{m n p} \partial_{q} \phi>,
$$

where $G_{m n p q} \equiv \partial_{m} C_{n p q}-\partial_{n} C_{m p q}+\partial_{p} C_{m n q}-\partial_{q} C_{m n p}$ is the field strength associated with the antisymmetric field $C_{m n p}$. This action was considered previously in reference [4] as a generalization to four dimensions of the Schwinger model in two dimensions. This action is invariant, up to a surface term, under the abelian gauge transformations

$$
\delta_{\xi} C_{m n p}=\partial_{m} \xi_{n p}+\partial_{n} \xi_{p m}+\partial_{p} \xi_{m n}, \quad \delta_{\xi} \phi=0 .
$$

and under the global transformation: $\delta \phi=$ constant. In $I_{M_{2}}$ we note that the coupling term is a BF topological term. This term preserves the number of degrees of freedom of the non-interacting terms (just the one connected with the massless (pseudo)scalar field), but change the physical characteristic of the spectrum (the (pseudo)scalar field becomes massive). This situation is like the one in three dimension with the topologically massive model where the topological Chern-Simons term changes the physical properties of the Maxwell field (which in three dimensions is a massless scalar) to a massive spin 1 theory. Other example of this situation, in four dimensions, occurs in 
the Cremmer-Scherk[16] or topologically massive model where the original fields are a massless scalar (one degree of freedom) and a photon with helicity 1 (two degrees of freedom) and the resulting theory with the topologiacal interaction term is a massive spin 1 excitation (three degrees of freedom).

The connection of $I_{M_{2}}$ and the massive (pseudo)scalar is straightforward: introducing $\lambda \equiv-\frac{1}{4} \epsilon^{m n p q} G_{m n p q}$ as the dual of the strenght field $G_{m n p q}$ into the action $I_{M 2}$, we observe that $\lambda$ plays the role of an auxiliary field, whose elimination through its equation of motion $(\lambda=-m \phi)$ leads to the action of a massive (pseudo)scalar field.

It is worth, at this point, to note that since $I_{M_{2}}$ depends only of derivatives of the scalar field, then its dual theory can be achieved, replacing $\partial_{m} \phi$ by $\frac{1}{2} l_{m}$ and adding a BF term: $\frac{1}{4} l_{m} \epsilon^{m n p q} \partial_{n} B_{p q}$ [17]. The dual action will be [4] [6]

$$
I_{d}=<-\frac{1}{2.4 !} G_{m n p q} G^{m n p q}-\frac{1}{2.3 !}\left(m C_{m n p}-H_{m n p}\right)\left(m C^{m n p}-H^{m n p}\right)>,
$$

where $H_{m n p}=\partial_{m} B_{n p}+\partial_{n} B_{p m}+\partial_{p} B_{m n}$ is the field strength of the antisymmetric field $B_{m n}$, which was introduced in the $\mathrm{BF}$ term. This action describes the interaction of open membranes whose boundaries are closed strings [18] and is invariant under the following gauge transformations

$$
\delta C_{m n p}=\partial_{m} \xi_{n p}+\partial_{n} \xi_{p m}+\partial_{p} \xi_{m n}, \quad \delta B_{m n}=\partial_{m} \lambda_{n}-\partial_{n} \lambda_{m}-m \xi_{m n} .
$$

The $\xi$ gauge transformation allows us gauged away the antisymmetric field $B_{m n}$, leading to the massive antisymmetric field $C_{m n p}$ action.

Let us, now, see that $I_{M_{2}}$ is locally equivalent to $I_{M 1}$ on-shell. The equations of motion for $I_{M 2}$ can be written, suggestively, as

$$
\begin{aligned}
& \epsilon^{m n p q} \partial_{q}\left(m C_{m n p}-\epsilon_{m n p r} \partial^{r} \phi\right)=0, \\
& \epsilon^{m n p q} \partial_{q}\left(m \phi-\frac{1}{3 !} \epsilon^{r s t u} \partial_{r} C_{s t u}\right)=0 .
\end{aligned}
$$

These equations, are just the result of aplying the differential operator $\epsilon^{m n p q} \partial_{q}$ to the equations of motion derived from $I_{M 1}$ : eqs. (四) and (5). From here the local equivalence can be stated: equations (10) and (11) can be written locally as

$$
m C_{m n p}-\epsilon_{m n p r} \partial^{r} \phi=\partial_{m} \lambda_{n p}+\partial_{n} \lambda_{p m}+\partial_{p} \lambda_{m n}
$$

and

$$
m \phi-\frac{1}{3 !} \epsilon^{m n p q} \partial_{m} C_{n p q}=\text { constant }
$$


Now, the right sides of (12) and (13) correspond, respectively, to gauge changes in $C_{m n p}$ and $\phi$. So those sides are gauge equivalent to zero, leaving us with equations (雨) and (5). In other words the physical relevant solutions of both models are the same, at the local level. Equivalently, closed 0 and 3 -forms $\left(\partial_{m} \phi=0=\partial_{[m} C_{n p q]}\right)$ are always solutions of the equations (10) and (11), while they become trivial in the model described by $I_{M 1}$. Locally, this closed forms are always exact and then the gauge invariance allow us to gauge them away. Globally, this will depend on the cohomological structure of the base manifolds and both models may differ in their space of solutions. We will see later that this topological dependence will appear explicitly in the partition function.

Proceeding with the comparison we will show the canonical equivalence for these two theories on cohomological trivial region of space-time. For the non-gauge invariant action $I_{M 1}$, the canonical hamiltonian density is found to be

$$
\mathcal{H}_{1}=\frac{1}{2.3 !} m^{2} C_{i j k} C_{i j k}+\frac{1}{2} m^{2} \phi^{2}+\frac{1}{2} \partial_{i} \phi \partial_{i} \phi,
$$

subject to the set of second class constraints

$$
\psi=0 \quad \text { and } \quad \psi_{i j k}=\pi_{i j k}-\frac{1}{3 !} m \epsilon_{i j k} \phi
$$

where $\pi$ and $\pi_{i j k}$ are the canonical conjugated momenta associated to $\phi$ and $C_{i j k}$, respectively.

On the other hand, for the gauge invariant action $I_{M 2}$, we have

$$
\mathcal{H}_{2}=3 \pi_{i j k} \pi_{i j k}+\frac{1}{2} \partial_{i} \phi \partial_{i} \phi+\frac{1}{2}\left[\pi-\frac{m}{3 !} \epsilon^{i j k} C_{i j k}\right]^{2},
$$

subject to the first class constraints

$$
\theta_{j k}=\partial_{i} \pi_{i j k}-\frac{1}{3 !} m \epsilon^{i j k} \partial_{i} \phi
$$

These constraints are doubly reducible, i.e., there are functions $Z_{k}^{i j}(x, y)$ and $Z_{k}(x, y)$ such that $\int d y Z_{k}^{i j}(x, y) \theta_{i j}(y)=0$ and $\int d y Z^{k}(x, y) Z_{k}^{i j}(x, z)=0$. This fact must be taken into account when we want the gauge freedom to be fixed.

It is straightforward to show that these two hamiltonian densities are related by linear combinations of the set of second class constraints of $\mathcal{H}_{1}$, 
reflecting the canonical equivalence:

$$
\mathcal{H}_{2}=\mathcal{H}_{1}+3 \psi_{i j k}\left[\psi_{i j k}+\frac{1}{3} m \epsilon_{i j k} \phi\right]+\frac{1}{2} \psi\left[\psi-\frac{1}{3} m \epsilon_{i j k} C_{i j k}\right] .
$$

This situation is similar to those theories which involve a mass mechanism through topological coupling [11 [9] 12]. Moreover, we can see that $I_{M 1}$ is a gauge fixed version of the gauge invariant action $I_{M 2}$. In fact, we note that $\theta_{i j}=\partial_{k} \psi_{i j k}$ can be considered as the first class constraints and $\psi=0$ as the gauge fixing condition $[$. In the latter case the first order hamiltonian results to be $\mathrm{H}_{2}$. The canonical procedure continues at this point and then the quantum equivalence is clear.

Now, in order to touch further aspects it is worth looking at the equivalence through the following master action

$$
\begin{aligned}
I_{M}= & <-\frac{1}{2.3 !} m^{2} a_{m n p} a^{m n p}-\frac{1}{2 !} m^{2} \psi^{2}+\frac{1}{4 !} m \epsilon^{m n p q} \psi G_{m n p q} \\
& +\frac{1}{3 !} m \epsilon^{m n p q}\left(a_{m n p}-C_{m n p}\right) \partial_{q} \phi>.
\end{aligned}
$$

Independent variations in $a_{m n p}, \psi, C_{m n p}$ and $\phi$ lead to the following equations of motion

$$
\begin{aligned}
& a^{m n p}=\frac{1}{m} \epsilon^{m n p q} \partial_{q} \phi, \\
& \psi=\frac{1}{4 ! m} \epsilon^{m n p q} G_{m n p q}, \\
& \epsilon^{m n p q} \partial_{m}(\psi-\phi)=0
\end{aligned}
$$

and

$$
\epsilon^{m n p q} \partial_{q}\left(a_{m n p}-C_{m n p}\right)=0 .
$$

Replacing the expressions for $a_{m n p}$ and $\psi$ given by eqs. (20) and (21) into $I_{M}$, the gauge invariant action $I_{M 2}$ is obtained. On the other hand, the solutions of the equations of motion (22) and (23) are

$$
\phi-\psi=\omega, \quad C_{m n p}-a_{m n p}=\Omega_{m n p},
$$

where $\omega$ and $\Omega_{m n p}$ are 0 and 3-closed forms, respectively. Locally, we can set

$$
\omega=\text { constant }, \quad \Omega_{m n p} \equiv L_{m n p}=\partial_{m} l_{n p}+\partial_{n} l_{p m}+\partial_{p} l_{m n}
$$

\footnotetext{
${ }^{1} \psi_{i j k}$ and $\partial_{i} \psi_{i j k}$ have the same information in three space dimensions
} 
and substituting into $I_{M}$, we obtain the following "Stüeckelberg" type action

$$
\begin{aligned}
I_{s}= & <-\frac{1}{2.3 !} m^{2}\left(C_{m n p}-L_{m n p}\right)\left(C^{m n p}-L^{m n p}\right)-\frac{1}{2} m^{2}(\phi-\omega)^{2} \\
& +\frac{1}{4 !} m \epsilon^{m n p q}(\phi-\omega) G_{m n p q}>
\end{aligned}
$$

This action is invariant under

$$
\delta_{\xi} C_{m n p}=\partial_{m} \xi_{n p}+\partial_{n} \xi_{p m}+\partial_{p} \xi_{m n}, \quad \delta_{\xi} l_{m n}=\xi_{m n}
$$

which allow us to gauge away the $l_{m n}$ field and recover $I_{M 1}$ (we have redefined $\phi-\omega$ as $\phi$ since $\omega$ is a constant). In this way, the local equivalence is also stated. On the other hand, we can consider $\psi=\phi-\omega$ and $a_{m n p}=$ $C_{m n p}-\Omega_{m n p}$ as general solutions in order to obtain the following gauge invariant action

$$
\begin{aligned}
\bar{I}_{M}= & <-\frac{1}{2.3 !} m^{2}\left(C_{m n p}-\Omega_{m n p}\right)\left(C^{m n p}-\Omega^{m n p}\right)-\frac{1}{2 !} m^{2}(\phi-\omega)^{2} \\
& +\frac{1}{4 !} m \epsilon^{m n p q}(\phi-\omega) G_{m n p q}-\frac{1}{3 !} m \epsilon^{m n p q} \Omega_{m n p} \partial_{q} \phi>.
\end{aligned}
$$

This action is global and locally equivalent to $I_{M 2}$, so the topological sectors, not present in $I_{M 1}$, are now included. Indeed, the equations of motion which are obtained after performing independent variations on $C_{m n p}, \Omega_{m n p}, \phi$ and $\omega$ in $\bar{I}_{M}$ are

$$
\begin{aligned}
& m\left(C^{m n p}-\Omega^{m n p}\right)-\epsilon^{m n p q} \partial_{q}(\phi-\omega)=0 \\
& m\left(C^{m n p}-\Omega^{m n p}\right)-\epsilon^{m n p q} \partial_{q} \phi=0
\end{aligned}
$$

and

$$
\begin{aligned}
& m(\phi-\omega)-\frac{1}{3 !} \epsilon^{m n p q} \partial_{m} C_{n p q}+\frac{1}{3 !} \epsilon^{m n p q} \partial_{m} \Omega_{n p q}=0 \\
& m(\phi-\omega)-\frac{1}{3 !} \epsilon^{m n p q} \partial_{m} C_{n p q}=0,
\end{aligned}
$$

from which is easily deduced that $\omega$ and $\Omega_{m n p}$ are closed 0 and 3-forms, respectively, i.e. $\epsilon^{m n p q} \partial_{q} \omega=0=\epsilon^{m n p q} \partial_{m} \Omega_{n p q}$. Taking into account this last result and applying the differential operator $\epsilon^{m n p q} \partial_{q}$ on the set first order differential equations given by (30), the equations of motion for the gauge 
invariant action $I_{M 2}$ are obtained (equations (10) and (11)). We can say that $\bar{I}_{M}$ is the correct modification to $I_{M_{1}}$ in order to include the, originally missing, topological sectors. This aspect will be important when interactions are to be considered. Moreover, we can eliminate $\phi$ and $C_{m n p}$ to achieve

$$
\bar{I}_{M 1}=I_{M 1[a, \psi]}-I_{t o p[\omega, \Omega]},
$$

where

$$
I_{t o p[\omega, \Omega]}=<\frac{1}{3 !} m \epsilon^{m n p q} \Omega_{m n p} \partial_{q} \omega>
$$

is the $\mathrm{BF}$ term for the topological coupling between 0 and 3 -forms in four dimensions. In other direction, if we couple a source to $\phi$ and $C_{m n p}$ only, in (28), it is easy to see that the sum of any solution of the inhomogeneous equations of $I_{M_{1}}$ with any solution of the inhomogeneous equation of the BF theory is a solution of the inhomogeneous equation of $I_{M_{2}}$.

From (32) we have that the partition funtions of $I_{M 1}$ and $I_{M 2}$ differ by a topological factor.

$$
Z_{M 2}=Z_{t o p} Z_{M 1}
$$

This topological factor is associated with the topologiacal sectors not present in the space of solutions of $I_{M 1}$. In general, on manifolds with non trivial topological structure $Z_{t o p} \neq 1$. Only when the manifold has a trivial structure, we will have $Z_{\text {top }} \equiv 1$, reflecting the local and global equivalence (In the cases of duality equivalence taking into account global aspects see references [19] [20]).

Finally, we will construct the supersymmetric formulation of the gauge invariant action for the massive axion. For this proposal, we need (anti)chiral superfields $\left(\Phi\right.$ and $\left.\Phi^{+}\right)$and a superfield $V$ whose supersymmetric field strength is $G=-\frac{1}{2}(D D+\bar{D} \bar{D}) V$, where $(D D V) \bar{D} \bar{D}$ is (anti) chiral superfield. This later superfield describes the $C_{m n p}$ in a supersymmetric way 21]. The supersymmetric action is

$$
\begin{aligned}
I & =\frac{1}{16} \int d^{4} x d^{2} \theta d^{2} \bar{\theta} G^{2}+\frac{1}{2} \int d^{4} x d^{2} \theta d^{2} \bar{\theta} \Phi \Phi^{+} \\
& +\frac{1}{4} m \int d^{4} x d^{2} \theta \Phi \bar{D} \bar{D} V+\frac{1}{4} m \int d^{4} x d^{2} \bar{\theta} \Phi^{+} D D V
\end{aligned}
$$

where the last two terms are the supersymmetric extension of the topological coupling. In components (using Majorana spinors), this action is written 
down as

$$
\begin{aligned}
I & =-\frac{1}{2} \partial_{m} A \partial_{m} A-\frac{1}{2} \partial_{m} B \partial_{m} B+\frac{1}{2}\left(\partial_{m} v_{m}\right)^{2}+\frac{1}{2} d^{2}-\frac{i}{2} \bar{\lambda} \gamma^{m} \partial_{m} \lambda \\
& -\frac{1}{2} \partial_{m} a \partial_{m} a-\frac{1}{2} \partial_{m} \phi \partial_{m} \phi-\frac{i}{2} \bar{\chi} \gamma^{m} \partial_{m} \chi+\frac{1}{2} f^{2}+\frac{1}{2} g^{2} \\
& -m\left[\phi \partial_{m} v_{m}+a d+i \bar{\chi} \gamma_{5} \lambda-(f B-g A)\right],
\end{aligned}
$$

where $\left(A, B, v^{m} \equiv \frac{1}{3 !} \epsilon^{m n p q} C_{n p q}, d, \lambda\right)$ and $(a, \phi, \chi, f, g)$ are the components of $\mathrm{V}$ and $\Phi$ multiplets, respectively. This action describes 8 bosonic and 8 fermionic degrees of freedom and is invariant under the off-shell supersymmetric transformations

$$
\begin{aligned}
\delta A & =i \bar{\epsilon} \lambda, \quad \delta B=i \bar{\epsilon} \gamma_{5} \lambda \\
\delta v & =i \bar{\epsilon} \gamma^{m} \lambda, \quad \delta d=i \bar{\epsilon} \gamma_{5} \gamma^{m} \partial_{m} \lambda \\
\delta \lambda & =\epsilon(\partial . v)+\gamma_{5} \epsilon d+\gamma^{m} \epsilon \partial_{m} A-\gamma_{5} \gamma^{m} \epsilon \partial_{m} A
\end{aligned}
$$

and

$$
\begin{aligned}
\delta a & =i \bar{\epsilon} \chi, \quad \delta \phi=i \bar{\epsilon} \gamma_{5} \chi \\
\delta \chi & =\left[\partial_{m} a-\gamma_{5} \partial_{m} \phi\right] \gamma^{m} \epsilon+\left[f+\gamma_{5} g\right] \epsilon \\
\delta f & =i \bar{\epsilon} \gamma^{m} \partial_{m} \chi, \quad \delta g=i \bar{\epsilon} \gamma_{5} \gamma^{m} \partial_{m} \chi
\end{aligned}
$$

Note that the $a, A$ and $B$ bosonic fields also have the same mass as the axion field, as can be seen after eliminating the $g, d$ and $f$ auxiliary fields, just as the dilaton acquire mass in the linear multiplet, in the supersymmetric formulation for the Cremmer-Sherk action 22

Sumarizing, we have seen that a gauge invariant description for massive axions is possible. This gauge invariant description is (locally)equivalent to the non-gauge invariant proposal. The supersymmetric formulation was given. A complete BRST analysis of the gauge invariant model considered in this paper is under consideration.

\section{ACKNOWLEDGEMENTS}

One of the authors (AK) would like to thank to the Consejo de Desarrollo Científico y Humanístico de la Universidad de los Andes(CDCHT-ULA) by institutional support under project C-862-97. 


\section{REFERENCES}

\section{References}

[1] R. Kallosh, A. Linde, D. Linde and L. Susskind, Phys. Rev. D52 (1995) 912.

[2] C.P. Burgess, J.P. Derendinger, F. Quevedo and M. Quiros, Phys. Lett. B348 (1995) 428.

[3] A. Aurilia and Y. Takahashi, Phys. Rev. D23 (1981) 1752.

[4] A. Aurilia, Y. Takahashi and P.K. Townsend, Phys. Lett. B95 (1980) 265.

[5] T. L. Curtright and P. O. Freund, Nucl. Phys. B172 (1980) 413.

[6] S. G. Rajeev, "Duality and Gauge Invariance" preprint MIT-CTP 1335 (unpublished).

[7] P.K. Townsend,K. Pilch and P. van Nieuwenhuizen, Phys. Lett. B136 (1984) 38; S. Deser and R. Jackiw, Phys. Lett. B139 (1984) 371.

[8] S. Deser, R. Jackiw and S. Tempelton, Ann. Phys. 140 (1982) 372; (E) 185 (1988) 406.

[9] P.J. Arias and L. Leal, Phys. Lett. B404 (1997) 49.

[10] T. J. Allen, M. Bowick and A. Lahiri, Mod. Phys. Lett. A6 (1991) 559.

[11] R. Gianvittorio, A.Restuccia and J. Stephany, Mod. Phys. Lett. A6 (1991) 2121.

[12] P.J. Arias and A. Restuccia, Phys. Lett. B347, (1995) 241.

[13] J. Stephany, Phys. Lett. B390 (1997) 128.

[14] E.M.Prodanov and S.Sen, Phys. Rev. D59 (1999) 065019.

[15] F. Quevedo, "Duality Beyond Global Symmetries: The Fate of the $B_{\mu \nu}$ Field" hep-th 9506081 
[16] E. Cremmer and J. Sherk, Nucl. Phys. B72 (1974) 117; M. Kalb and P. Ramond, Phys. Rev. D9 (1974) 2273.

[17] H. Nicolai and P.K. Townsend, Phys. Lett. B98, (1981) 257.

[18] A. Aurilia and Y. Takahashi, Progress of Theo. Phys. 66 (1981) 693.

[19] T. J. Allen, Nucl. Phys. B395 (1993) 185.

[20] M. I. Caicedo, I. Martin and A. Restuccia, "Duality on Higher Order U(1) Bundles" hep-th 9701010.

[21] S.J. Gates, Nucl. Phys. B184 (1981) 381.

[22] W. Siegel, Phys. Lett. B85 (1979) 333. 